\title{
Control of broad-area vertical-cavity surface emitting laser emission by optically induced photonic crystals
}

\author{
Bernd Terhalle, ${ }^{1, a)}$ Neal Radwell, ${ }^{2}$ Patrick Rose, ${ }^{1}$ Cornelia Denz, ${ }^{1}$ and Thorsten Ackemann ${ }^{2}$ \\ ${ }^{1}$ Institut für Angewandte Physik and Center for Nonlinear Science (CeNoS), Westfälische Wilhelms- \\ Universität Münster, Corrensstraße 2/4, 48149 Münster, Germany \\ ${ }^{2}$ SUPA and Department of Physics, University of Strathclyde, Glasgow, G40NG Scotland, United Kingdom
}

(Received 7 August 2008; accepted 16 September 2008; published online 17 October 2008)

\begin{abstract}
We control the emission properties of a broad-area vertical-cavity surface emitting laser by coupling it to an external feedback cavity containing a photorefractive crystal with an optically induced photonic lattice. The periodic modulation of the refractive index serves as a tunable filter and enables the dynamic suppression of unwanted spatial instabilities and modes, as originally suggested by Gomila et al. [Phys. Rev. Lett. 92, 253904 (2004)]. (C) 2008 American Institute of Physics.
\end{abstract}

[DOI: 10.1063/1.2996257]

In recent years the control of light generation and propagation by periodic structures which induce a photonic bandgap in one, two, or potentially three spatial dimensions found considerable interest. ${ }^{1-3}$ Due to the tremendous advances in nanofabrication of semiconductor structures, it seems to be especially attractive to control semiconductor laser emission by combining them with photonic structures. Indeed several demonstrations are known for edge emitting ${ }^{4,5}$ as well as for vertical-cavity schemes. ${ }^{6-8}$ However, in these configurations, the holes incorporated into the semiconductor increase optical losses due to scattering and carrier losses due to increased recombination. Hence, in all schemes demonstrated until now, emission takes place in defects of the photonic crystal. In contrast, there are theoretical proposals ${ }^{9,10}$ to control the spatial complexity inherent to broad-area nonlinear optical resonators and lasers by incorporating homogeneous intracavity photonic crystals. This method seems to be particularly suited for broad-area vertical-cavity surface emitting lasers (VCSELs), which have a transverse extension much larger than their length. As a result, they are prone to the excitation of transverse spatial modes and their brilliance is limited although they would be desirable for applications in material processing, medical therapies, and laser pumping because of their nearly symmetrical beam profile (e.g., Ref. 11). Some broad-area VCSELs were shown to emit superpositions of Fourier modes (approximate plane waves) with a rather defined spatial wavenumber ${ }^{12-14}$ which opens up the possibility for a control by a photonic bandgap structure. The basic idea is that the propagation of unwanted off-axis plane waves with a certain wave vector can be inhibited by a one- or multidimensional photonic structure.

In this letter, we demonstrate the proof of principle of this technique by showing the inhibition of a transverse wavevector component $k_{0}$ by a $1 d$ photonic structure with a wavenumber $k_{B}=2 k_{0} \sin \theta$ in the transverse plane. The photonic structure is realized in a photorefractive crystal using the optical induction technique. ${ }^{15}$ Such light-induced photonic structures were demonstrated to be very suitable for studying principles of linear and nonlinear optics in periodic media before (see, e.g., Ref. 16 and references therein). In particular, the stabilization of counterpropagating solitons by

${ }^{\text {a)} E l e c t r o n i c ~ m a i l: ~ b e r n d . t e r h a l l e @ u n i-m u e n s t e r . d e . ~}$
$1 d$ photonic lattices ${ }^{17}$ shows the potential of optically induced photonic lattices to control spatiotemporal nonlinear dynamics.

The experimental setup is shown schematically in Fig. 1(a). We use VCSELs similar to the ones described in Refs 12 and 13 with an aperture size of $40 \mu \mathrm{m}$ and add an external cavity containing a $20 \mathrm{~mm}$ long photorefractive $\mathrm{Sr}_{0.60} \mathrm{Ba}_{0.40} \mathrm{Nb}_{2} \mathrm{O}_{6} \quad$ (SBN:Ce) crystal in which a onedimensional photonic lattice is induced by interference of two ordinarily polarized plane waves with a power of $150 \mu \mathrm{W}$ each. The interference pattern is created in a MachZehnder-type configuration containing two half wave plates and a polarizer to adjust the intensities of the two beams. The VCSEL is operated at a temperature of $10^{\circ} \mathrm{C}$ and emits at a wavelength of $\lambda \approx 780 \mathrm{~nm}$. The output is collected by a collimating lens and subsequently imaged onto a highly reflective feedback mirror using a second lens. The cavity configuration corresponds to a telescope providing self-imaging feedback and thus preserving the high Fresnel number of the VCSEL. ${ }^{18}$ After the second lens, a tilted plane wave emerging from the VCSEL is again a tilted plane wave but the tilt angle is decreased and hence the period of the interference

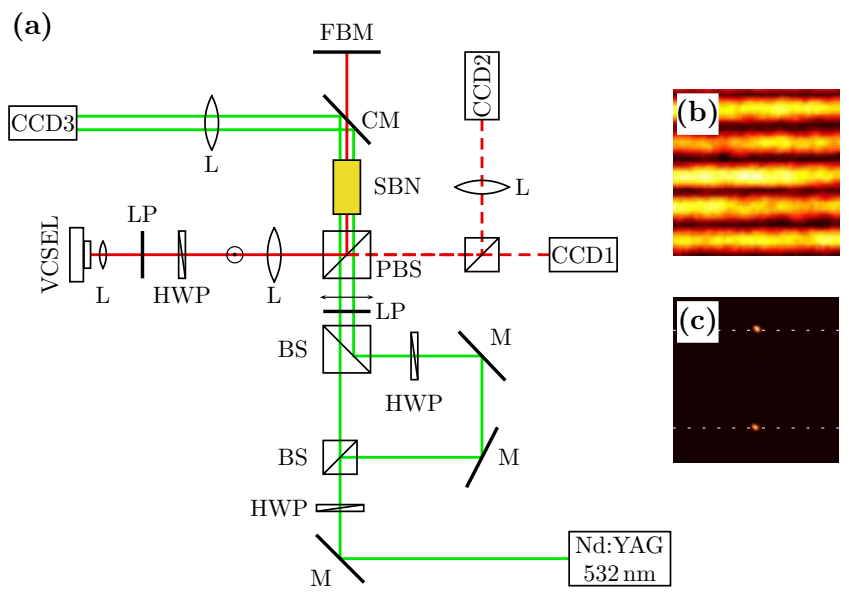

FIG. 1. (Color online) Schematic experimental setup; L: lens; LP: polarizer; HWP: half wave plate; M: mirror; BS: beamsplitter; PBS: polarizing beamsplitter at $780 \mathrm{~nm}$; CM: cold mirror (HR at $532 \mathrm{~nm}$, HT at $780 \mathrm{~nm}$ ); FBM: feedback mirror; CCD: camera; (b) intensity distribution of the lattice wave with a period of $d=46 \mu \mathrm{m}$; (c) Fourier space image of the lattice wave containing two bright spots marking the borders of the first Brillouin zone. 

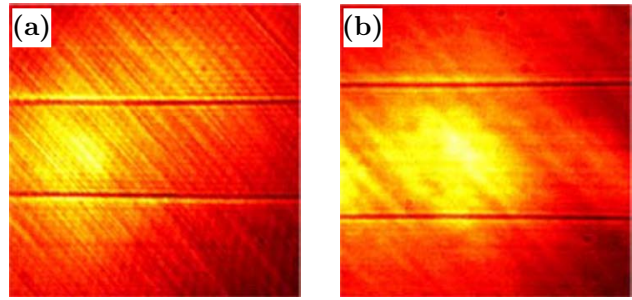

FIG. 2. (Color online) Experimentally observed Brillouin zones for a onedimensional stripe pattern (period $d=16 \mu \mathrm{m}$ ) at different probe beam wavelengths; (a) $532 \mathrm{~nm}$; (b) $780 \mathrm{~nm}$.

pattern is increased. The focal lengths of the lenses are chosen such that the the length scale of the structure in the VCSEL to be controlled $(2-3 \mu \mathrm{m})$ is mapped to a scale of the order of some tens of micrometers, which can be conveniently written within the photorefractive crystal. The polarizing beamsplitter ensures the extraordinary polarization necessary to exploit the strong electro-optic coefficient $r_{33}$ of the crystal. At the same time, it enables the imaging onto the two cameras CCD1 and CCD2 by slightly tilting the incoming polarization using a half wave plate. Behind the crystal, the cavity contains a dichroitic mirror to remove the green light of the lattice beams from the feedback path. The removed light is then used to image the lattice wave onto the camera CCD3.

Figure 1(b) shows the corresponding periodic intensity distribution at the front face of the crystal. According to the two plane waves used for creation of the interference pattern, the Fourier image [Fig. 1(c)] consists of two bright spots which also determine the borders of the first Brillouin zone of the induced lattice. For illustration, the Brillouin zone edges are additionally marked by two dashed lines in Fig. 1(c). Wave propagation at these transverse wave vectors is forbidden as a result of multiple Bragg reflections inside the lattice and leads to dark lines in the transmission spectrum. ${ }^{3}$ The experimentally observed Brillouin zone pictures for a one-dimensional stripe pattern are shown in Fig. 2 for two different wavelengths. In both cases, the dark lines marking the borders of the first Brillouin zone where the Bragg condition is satisfied are clearly visible. Therefore, the induced structure can be used to control the feedback and selectively suppress the propagation of the corresponding off-axis modes.

Due to the reconfigurability of the optically induced lattices, the filtering can be dynamically tuned to another transverse wavenumber by simply changing the lattice constant or even turned off completely by erasing the lattice using homogeneous white light illumination. The experimental results of this dynamic control of off-axis modes with a specific transverse wave number are summarized in Fig. 3. The VCSEL is driven close to threshold $(13.15 \mathrm{~mA})$ such that there is no laser emission without feedback. However, including the feedback path, it starts lasing in the modulated state shown in Figs. 3(a) and 3(b). In the far field [Fig. 3(b)], the emission contains an on-axis spot centered around wavenumber zero together with a set of off-axis modes. The corresponding real space image consists of a localized spot in addition to a modulated stripe pattern as shown in Fig. 3(a).

By applying an electric field of $1 \mathrm{kV} / \mathrm{cm}$ and the writing beams, a photonic lattice with a period of $46 \mu \mathrm{m}$ is induced inside the photorefractive crystal. As demonstrated in Figs.
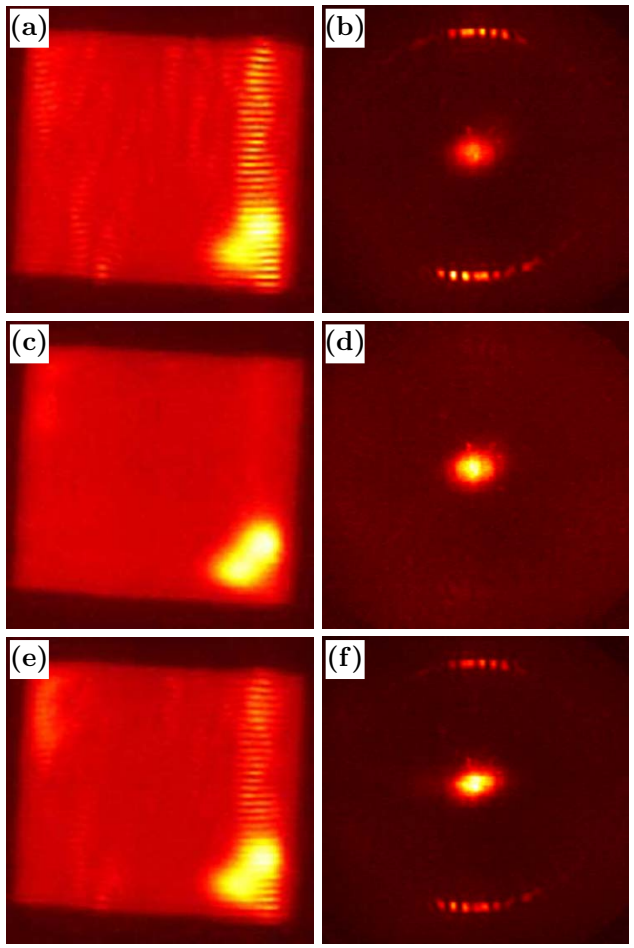

FIG. 3. (Color online) VCSEL output in real space (left column) as well as in Fourier space (right column). [(a) and (b)] Output without an induced lattice; [(c) and (d)] output with lattice; [(e) and (f)] output after erasing the lattice with homogeneous white light illumination.

3(c) and 3(d) the corresponding transverse wavenumbers can be suppressed this way. The far field now consists of one single spot at wavenumber zero [Fig. 3(d)], while in the near field only the well defined localized spot remains [Fig. 3(c)]. It is important to note that the crystal is almost insensitive in the infrared region and any influence of the VCSEL beam on the lattice can therefore be neglected. After the grating is written, the lattice-writing beams can be switched off and the control still works because of the long lifetime of the photorefractive grating (several days for ambient light levels typical for a laboratory). Active erasure of the grating and thus dynamic tunability of our control technique are achieved by homogeneously illuminating the crystal with white light. As a result, the filtering vanishes and the VCSEL returns to the same modulated state [see Figs. 3(e) and 3(f)] as in Figs. 3(a) and $3(\mathrm{~b})$. We note that in the present scheme, feedback strength and thus control range are limited by the Fresnel losses at the crystal. Therefore, the use of an antireflection coated crystal will certainly be beneficial in a future device application. Another very important feature of photorefractive SBN crystals is their strong polarization anisotropy. ${ }^{19}$ Due to the small electro-optic coefficient $r_{13}$, an ordinarily polarized beam is hardly affected while an extraordinarily polarized beam $\left(r_{33} \gg r_{13}\right)$ gets strongly influenced by the induced lattice. Therefore, changing the polarization of the probe beam offers an additional control technique, as shown in Fig. 4. As before, the VCSEL is operated at $10{ }^{\circ} \mathrm{C}$ and the current of $13.24 \mathrm{~mA}$ is again close to threshold. As demonstrated in Ref. 12 the approximate polarization isotropy of VCSELs is broken for off-axis waves, leading to horizontal polarization for the investigated off-axis states. By placing a half wave plate behind the polarizing beam splitter, the polarization of the VCSEL beam can be turned from extraordi- 

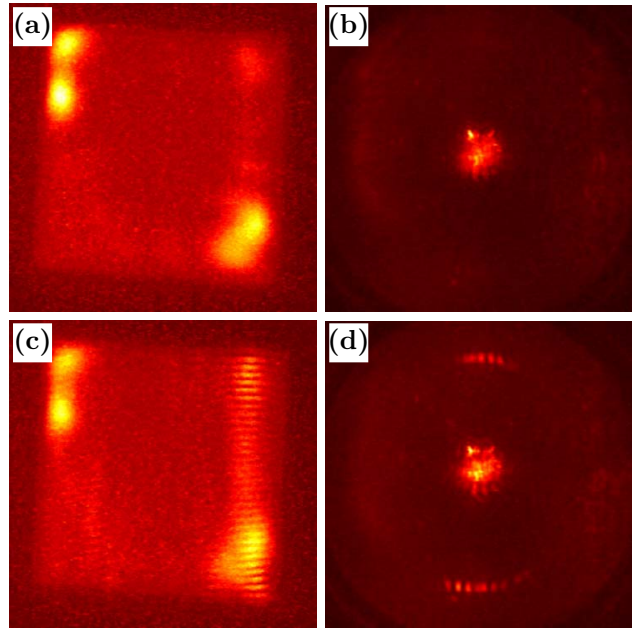

FIG. 4. (Color online) Emission control by changing the polarization state of the beam propagating in the feedback loop. [(a) and (b)] Extraordinary polarization; [(c) and (d)] ordinary polarization.

nary to ordinary, and thus the selective off-axis mode filtering can be controlled. In the case of extraordinary polarization, these modes are suppressed and the VCSEL lases at three localized spots [Fig. 4(a)]. The far field pattern, Fig. 4(b), consequently contains one central spot similar to the one shown in Fig. 3(b). However, if the polarization is turned to ordinary, the suppressed modes reappear and the VCSEL again operates in the modulated state [Figs. 4(c) and 4(d)]. Note that the lattice is still present and the absence of the filtering capability is only caused by the polarization anisotropy of the SBN crystal.

In conclusion, we have demonstrated the control of broad-area vertical surface emitting lasers by coupling them to an external cavity containing an optically induced photonic crystal, which enables the suppression of unwanted spatial instabilities and modes. Due to the reconfigurability of the periodic refractive index modulation, the filtering can be tuned to a specific transverse wavenumber or even turned off using homogeneous white light illumination. Moreover, the polarization anisotropy of the used SBN crystals has been shown to provide an additional control possibility. The dynamic reconfigurability is a particular useful feature of light- induced gratings but permanent control can be achieved by either a fixation of a light-induced grating by established techniques or by microfabrication of a periodic structure.

We acknowledge support by the British Council, the Royal Society, and the German Academic Exchange Service (DAAD) and are grateful to K. F. Huang for supplying the VCSEL devices, to M. Schulz-Ruhtenberg for help with the setup, and to G.-L. Oppo and W. J. Firth for useful discussions.

${ }^{1}$ J. Pendry, Science 285, 1687 (1999).

${ }^{2}$ R. De La Rue, Opt. Photonics News 17, 30 (2006).

${ }^{3}$ G. Bartal, O. Cohen, H. Buljan, J. W. Fleischer, O. Manela, and M. Segev, Phys. Rev. Lett. 94, 163902 (2005).

${ }^{4}$ L. Zhu, P. Chak, J. K. S. Poon, G. A. DeRose, A. Yariv, and A. Scherer, Opt. Express 15, 5966 (2007).

${ }^{5}$ S. A. Moore, L. O'Faolain, T. P. White, and T. F. Krauss, Opt. Express 16, 1365 (2008).

${ }^{6}$ A. J. Danner, J. J. Raftery, Jr., N. Yokouchi, and K. D. Choquette, Appl. Phys. Lett. 84, 1031 (2004).

${ }^{7}$ K.-H. Lee, J.-H. Baek, I.-K. Hwang, Y.-H. Lee, G.-H. Lee, J.-H. Ser, H.-D. Kim, and H.-E. Shin, Opt. Express 12, 4136 (2004).

${ }^{8}$ E. Miyai, K. Sakai, T. Okano, W. Kunishi, D. Ohnishi, and S. Noda, Nature (London) 441, 946 (2006).

${ }^{9}$ D. Gomila, R. Zambrini, and G.-L. Oppo, Phys. Rev. Lett. 92, 253904 (2004).

${ }^{10}$ D. Gomila and G.-L. Oppo, Phys. Rev. E 72, 016614 (2005).

${ }^{11}$ M. Grabherr, R. Jäger, M. Miller, C. Thalmaier, J. Heerlein, R. Michalzik, and K. J. Ebeling, IEEE Photonics Technol. Lett. 10, 1061 (1998).

${ }^{12}$ I. Babushkin, M. Schulz-Ruhtenberg, N. A. Loiko, K. F. Huang, and T. Ackemann, Phys. Rev. Lett. 100, 213901 (2008).

${ }^{13}$ M. Schulz-Ruhtenberg, I. V. Babushkin, N. A. Loiko, T. Ackemann, and K. F. Huang, Appl. Phys. B: Lasers Opt. 81, 945 (2005).

${ }^{14}$ S. P. Hegarty, G. Huyet, J. G. McInerney, and K. D. Choquette, Phys. Rev. Lett. 82, 1434 (1999)

${ }^{15}$ J. W. Fleischer, T. Carmon, M. Segev, N. K. Efremidis, and D. N. Christodoulides, Phys. Rev. Lett. 90, 023902 (2003).

${ }^{16}$ J. W. Fleischer, G. Bartal, O. Cohen, T. Schwartz, O. Manela, B. Freedman, M. Segev, H. Buljan, and N. K. Efremidis, Opt. Express 13, 1780 (2005).

${ }^{17}$ S. Koke, D. Träger, P. Jander, M. Chen, D. N. Neshev, W. Krolikowski, Y. S. Kivshar, and C. Denz, Opt. Express 15, 6279 (2007).

${ }^{18}$ Y. Tanguy, T. Ackemann, W. J. Firth, and R. Jäger, Phys. Rev. Lett. 100, 013907 (2008).

${ }^{19}$ B. Terhalle, A. S. Desyatnikov, C. Bersch, D. Träger, D. N. Neshev, L. Tang, J. Imbrock, Y. S. Kivshar, and C. Denz, Appl. Phys. B: Lasers Opt. 86, 399 (2007). 